\title{
A LOW-COST SPATIAL TOOL FOR TRANSFORMING FEATURE POSITIONS OF CAD-BASED TOPOGRAPHIC MAPPING
}

\author{
Maan HABIB $^{1^{*}}$, A'kif ALFUGARA², Biswajeet PRADHAN ${ }^{3}$ \\ ${ }^{1}$ Department of Civil Engineering, Al-Balqa Applied University, Al Salt, Jordan \\ ${ }^{2}$ Department of Surveying Engineering, Al al-Bayt university, Mafraq, Jordan \\ ${ }^{3}$ Faculty of Engineering and IT, Research Centre for Advanced Modelling and Geospatial Information, \\ Systems (CAMGIS), University of Technology Sydney, NSW, Australia
}

Received 14 May 2019; accepted 22 November 2019

\begin{abstract}
In fact, Computer Aided Design (CAD) offers powerful design tools to produce digital large scale topographic mapping that is considered the backbone for construction projects, urban planning and landscape architecture. Nowadays local agencies in small communities and developing countries are facing some difficulties in map to map transformation and handling discrepancies between the physical reality and represented spatial data due to the need for implementing high cost systems such as GIS and the experienced staff required. Therefore, the require for providing a low-cost tool based on the most common CAD system is very important to guarantee a quality and positional accuracy of features. The main aim of this study is to describe a mathematical relationship to fulfil the coordinate conversion between two different grid references applying two-dimensional conformal polynomial models built on control points and a least squares fitting algorithm. In addition, the automation of this model was performed in the Microsoft Visual Studio environment to calculate polynomial coefficients and convert the positional property of entities in AutoCAD by developing spatial CAD tool. To evaluate the proposed approach the extracted coordinates of check points from the interpolation surface are compared with the known ones.
\end{abstract}

Keywords: conformal transformation, least squares adjustment, polynomials, AutoCAD automation.

\section{Introduction}

Representation of the Earth's surface on a plane is a significant and complex topic of surveyor to produce topographic maps at the field scale in many applications in civil engineering. AutoCAD software is considered as a useful and easy tool for making digital large-scale engineering mapping in a vector model. The reference map provides general information about features location in accurate coordinate system.

The GIS system is applied to capture, store, retrieve, analyze and visualize spatial data with a view to support decision-making (Awange \& Kiema, 2013), while Computer Aided Design (CAD) is the use of computer systems to help in the creation, optimization, modification, or analysis of a design (Moreno \& Bazán, 2017). Combining data available in GIS and CAD requires new methodologies and models that merge real world phenomena while maintaining the details of entities for facilitating calculation and representation (Karimi \& Akinci, 2009). CAD users are seeking to utilize data exported from GIS and conversely, they are also creating attributed GIS layers within CAD files ready for direct use in the GIS system (Esri, 2011).

Geospatial data depict features from real world in terms of (a) their position with reference to an applied coordinate system, (b) their attributes that are unrelated to location, and (c) their topological relations that represent how they are related to each other (Burrough, R. McDonnell, R. A. McDonnell, \& Lloyd, 2015). The high level of the positional accuracy of objects is very significant, therefore they must be illustrated in a suitable coordinate reference system.

Georeferenced data are captured from various sources that usually reveals the data have different coordinate systems, therefore changing the coordinates must be applied to unify data references. Transformations between diverse

${ }^{*}$ Corresponding author. E-mail: maan.habib@bau.edu.jo 
frameworks are not just preliminary steps, but essential for GIS analysis (Chrisman, 1999). It indicates how observations captured from one sort of geometric model can be converted to another one, subject to specific constraints (Snyder, 1987). The angles in conformal conversion are correctly represented and scale is the same in all directions at any point (Thomas, 1952).

The transformation components of any point within the grid are generally determined by parameters of interpolation surface utilizing common stations in both systems (Janssen, 2009). The number of control stations and their spatial distribution play a very important role in the conversion process since they affect the accuracy of the mathematical models. Thus, redundant data points give the best polynomial coefficients by selecting good stations. Least squares approach is applied to estimate the most probable values of polynomial coefficients from redundant observations and determine the accuracy and reliability of measured and estimated quantities (Taşçi, 2009).

There are many commercial, PC-based CAD packages available for assisting in generating engineering drawings. One of the most known software is AutoCAD, which was developed by Autodesk Corporation to supply a flexible development platform for specialized design and applications. AutoCAD data are much more precise than GIS data on large-scale maps, but they often adopt coordinate systems different from that of GIS (Shi et al., 2016).

The main objective of this study is to develop a software tool in the Microsoft Visual Studio environment that can be applied to match DWG data from one coordinate system to another, with preserving all other properties or thematic elements without any change. Furthermore, this research provides a verification of the proposed approach by performing the transformation between two maps from various control stations using polynomial model and comparing the computed coordinates of check points with the rectangular coordinates derived from a map.

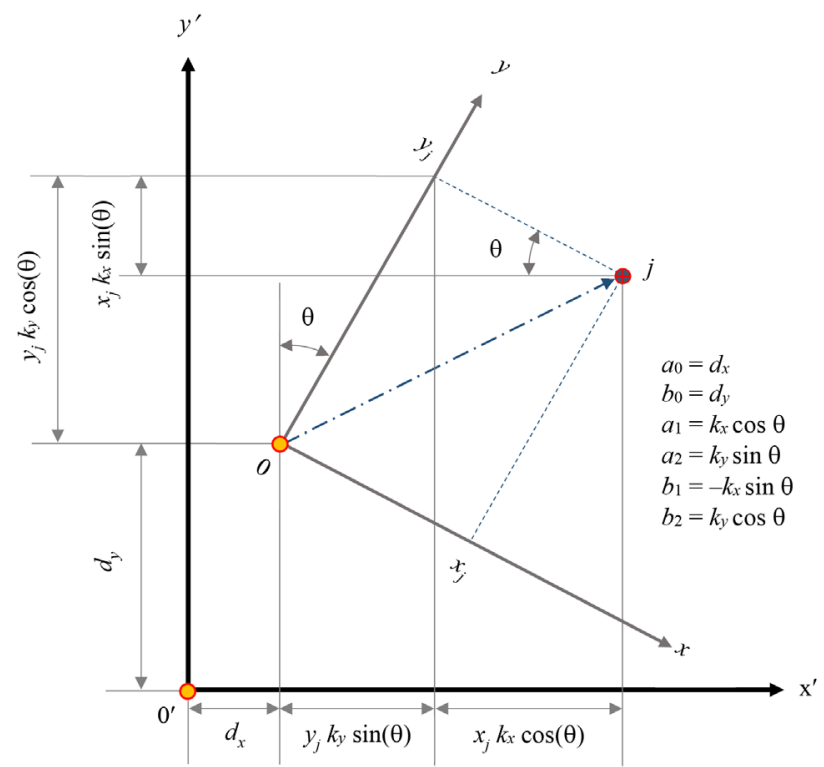

Figure 1. Two-dimensional coordinate transformation

\section{Mathematical approach}

A coordinate transformation is a mathematical procedure that matches between the coordinates of a point in one grid system with another (Maling, 1992). In general, a two-dimensional (2D) coordinate conversion can be split into combining axes rotations, origin shifts and a scale changes as shown in Figure 1. The most common one in surveying applications, and the only type dealt with in this study is conformal that preserve angles and thus the shape of objects. A transformation model is usually defined by polynomial functions of degree, $u$, with an infinite number of terms as follows (Herrault, Sheeren, Fauvel, Monteil, \& Paegelow, 2013):

$$
x^{\prime}=\sum_{i=0}^{u} \sum_{j=0}^{u-i} a_{i j} x^{i} y^{j}, y^{\prime}=\sum_{i=0}^{u} \sum_{j=0}^{u-i} b_{i j} x^{i} y^{j},
$$

where, $a_{i j}$ and $b_{i j}$ are the polynomial coefficients. Expanding Eq. (1) to the first power gives the same equations as in affine transformation:

$$
\begin{aligned}
& x^{\prime}=a_{0}+a_{1} x+a_{2} y ; \\
& y^{\prime}=b_{0}+b_{1} x+b_{2} y .
\end{aligned}
$$

The conformal mapping of coordinates using polynomials can be done by applying the Cauchy-Riemann conditions on (Deakin, 2004) previous model in Eq. (1).

$$
\frac{\partial x^{\prime}}{\partial x} \equiv \frac{\partial y^{\prime}}{\partial y}, \quad \frac{\partial x^{\prime}}{\partial y} \equiv-\frac{\partial y^{\prime}}{\partial x} \text {. }
$$

However, the relation between any two conformal projections can be defined by the following equation:

$$
z^{\prime}=f(z),
$$

where

$$
\begin{aligned}
& z^{\prime}=x^{\prime}+i y^{\prime} ; \\
& z=x+i y,
\end{aligned}
$$

$\left(x^{\prime}, y^{\prime}\right)$ and $(x, y)$ are rectangular coordinates of the two respective projections for the same point and $i$ is the imaginary number $\left(i^{2}=-1\right)$. In general, the function $f$ that describes the relationship between these coordinates is not known. Eq. (4) may be expanded in a Maclaurin's series as

$$
z^{\prime}=C_{0}+C_{1} z+C_{2} z^{2}+\ldots .
$$

The $C$ s are complex coefficients of the form

$$
C_{k}=a_{k}+i b_{k} \text {. }
$$

In any textbook on complex variables, it is shown that every polynomial in $\mathrm{z}$ is analytic (i.e. satisfies the CauchyRiemann conditions) at all points; therefore Eq. (4) represents a conformal transformation. Writing out the first few terms of Eq. (4) in full, then

$$
\begin{aligned}
& x^{\prime}+i y^{\prime}=\left(a_{o}+i b_{o}\right)+\left(a_{1}+i b_{1}\right)(x+i y)+ \\
& \left(a_{2}+i b_{2}\right)(x+i y)^{2}+\cdots .
\end{aligned}
$$

Or in a more generalized form (Pędzich, 2005) 


$$
x^{\prime}+i y^{\prime}=\sum_{k=0}^{u}\left(a_{k}+i b_{k}\right)(x+i y)^{k} .
$$

The number of the conformal polynomial coefficients, $m$, is related to the degree of polynomial and equals $m=$ $2 \times u+2$. The four terms up to first degree, $u=1$, represent the well-known similarity or Helmet's transformation. Terms beyond the first power represent what is known as conformal deformation. However, the fifth power conformal functions are

$$
\begin{aligned}
x^{\prime}= & a_{0}+a_{1} x-b_{1} y+ \\
& a_{2}\left(x^{2}-y^{2}\right)-2 b_{2} x y+ \\
& a_{3}\left(x^{3}-3 y^{2} x\right)+b_{3}\left(y^{3}-3 x^{2} y\right)+ \\
& a_{4}\left(x^{4}+y^{4}-6 x^{2} y^{2}\right)-b_{4}\left(4 x^{3} y-4 x y^{3}\right)+ \\
& a_{5}\left(x^{5}+5 y^{4} x-10 x^{3} y^{2}\right)-b_{5}\left(y^{5}+5 x^{4} y-10 x^{2} y^{3}\right) ; \\
y^{\prime}= & b_{0}+a_{1} y+b_{1} x+ \\
& 2 a_{2} x y+b_{2}\left(x^{2}-y^{2}\right)+ \\
& b_{3}\left(x^{3}-3 y^{2} x\right)-a_{3}\left(y^{3}-3 x^{2} y\right)+ \\
& a_{4}\left(4 x^{3} y-4 x y^{3}\right)+b_{4}\left(x^{4}+y^{4}-6 x^{2} y^{2}\right)+ \\
& a_{5}\left(y^{5}+5 x^{4} y-10 x^{2} y^{3}\right)+b_{5}\left(x^{5}+5 y^{4} x-10 x^{3} y^{2}\right) .
\end{aligned}
$$

Scale factor of a point and convergence of meridian for this conversion are calculated as follows (Vanicek \& Krakiwsky, 1987):

$$
\begin{aligned}
& K=\sqrt{\left(\frac{\partial x^{\prime}}{\partial x}\right)^{2}+\left(\frac{\partial y^{\prime}}{\partial x}\right)^{2}} \\
& \tan \theta=-\frac{\partial y^{\prime}}{\partial x} / \frac{\partial x^{\prime}}{\partial x}
\end{aligned}
$$

$$
\begin{aligned}
\frac{\partial x^{\prime}}{\partial x}= & a_{1}+2 a_{2} x-2 b_{2} y+3 a_{3}\left(x^{2}-y^{2}\right)-6 b_{3} x y+ \\
& 4 a_{4}\left(x^{3}-3 y^{2} x\right)-4 b_{4}\left(3 x^{2} y-y^{3}\right)+ \\
& 5 a_{5}\left(x^{4}+y^{4}-6 y^{2} x^{2}\right)-20 b_{5}\left(x^{3} y-y^{3} x\right) ;
\end{aligned}
$$

$$
\begin{aligned}
\frac{\partial y^{\prime}}{\partial x}= & b_{1}+2 a_{2} y+2 b_{2} x+3 b_{3}\left(x^{2}-y^{2}\right)+6 a_{3} x y+ \\
& 4 b_{4}\left(x^{3}-3 y^{2} x\right)+4 a_{4}\left(3 x^{2} y-y^{3}\right)+ \\
& 5 b_{5}\left(x^{4}+y^{4}-6 y^{2} x^{2}\right)+20 a_{5}\left(x^{3} y-y^{3} x\right) .
\end{aligned}
$$

\section{Determination of the transformation parameters}

A map to map transformation requires to estimate conversion parameters by applying a least squares approach through common points. The method of least squares is widely used to fit data to a mathematical model that is generally formulated as a Gauss-Markov model (Velsink, 2018). However, the accuracy of estimated transformation parameters is highly correlated to certain characteristics of the control points (Kutoglu \& Vaníček, 2006). In any case, extrapolation should be avoided and thus the common stations must surround any unknown coordinate points. Therefore, a transformation of first-degree this requires that an unknown point lie within a triangle formed by three known ones, but second-degree needs the station lie within a quadrilateral fixed by four control points (Vincenty, 1987).

Using least squares adjustment in the transformation problems gives the best estimation of parameters by analysing simultaneously all the common points (Greenfeld, 1997). The mathematical model of the parametric technique for least square adjustment in matrix form is (Mikhail \& Gracie, 1981)

$$
\underset{2 n \times 1}{V}+\underset{2 n \times m}{B} \cdot \underset{m \times 1}{\Delta}=\underset{2 n \times 1}{f},
$$

where, $V$ vector of observational residuals, $B$ numerical coefficients matrix of parameters, $\Delta$ vector of unknown parameters, $f$ vector of numerical constants, $\mathrm{n}$ number of common stations and $m$ number of the polynomial coefficients. The solution of observation equations as follows:

$$
\begin{aligned}
& \underset{m \times m}{N}=\underset{m \times 2 n}{B^{t}} \underset{2 n \times m}{B} ; \\
& \underset{m \times 1}{t}=\underset{m \times 2 n}{B^{t}} \cdot \underset{2 n \times 1}{f} ; \\
& \Delta=N^{-1} t .
\end{aligned}
$$

In writing the transformation equations, in the form given here, any redundant measurement admits to errors in system $(x, y)$, while system $\left(x^{\prime}, y^{\prime}\right)$ values are considered constant. The matrix form of Eq. (15) and (16) is:

$$
\begin{aligned}
& v_{x}=x^{\prime}-\left(a_{0}+a_{1} x-b_{1} y+a_{2}\left(x^{2}-y^{2}\right)-2 b_{2} x y+\cdots\right) \\
& v_{y}=y^{\prime}-\left(b_{0}+b_{1} x+a_{1} y+b_{2}\left(x^{2}-y^{2}\right)+2 a_{2} x y+\cdots\right) .
\end{aligned}
$$

By writing these equations for common points in the form $V+B \Delta=f$, then:

$$
\begin{aligned}
& v_{x_{i}}+a_{0}+a_{1} x_{i}-b_{1} y_{i}+a_{2}\left(x_{i}^{2}-y_{i}^{2}\right)-2 b_{2} x_{i} y_{i}+\cdots=x_{i}^{\prime} ; \\
& v_{y_{i}}+b_{0}+b_{1} x_{i}+a_{1} y_{i}+b_{2}\left(x_{i}^{2}-y_{i}^{2}\right)+2 a_{2} x_{i} y_{i}+\cdots=y_{i}^{\prime} ;
\end{aligned}
$$

$$
B=\left[\begin{array}{cccccccc}
1 & x_{1} & \left(x_{1}^{2}-y_{1}^{2}\right) & \ldots & 0 & -y_{1} & -2 x_{1} y_{1} & \ldots \\
0 & y_{1} & 2 x_{1} y_{1} & \ldots & 1 & x_{1} & \left(x_{1}^{2}-y_{1}^{2}\right) & \ldots \\
& & \ldots & & \ldots & \ldots & \\
& & \ldots & & \ldots & \ldots & \\
1 & x_{n} & \left(x_{n}^{2}-y_{n}^{2}\right) & \ldots & 0 & -y_{n} & -2 x_{n} y_{n} & \ldots \\
0 & y_{n} & 2 x_{n} y_{n} & \ldots & 1 & x_{n} & \left(x_{n}^{2}-y_{n}^{2}\right) & \ldots
\end{array}\right] ;
$$




$$
\begin{aligned}
\Delta^{t} & =\left[\begin{array}{llllllll}
a_{0} & a_{1} & \ldots & a_{5} & b_{0} & b_{1} & \ldots & b_{5}
\end{array}\right] \\
f^{t} & =\left[\begin{array}{llllll}
x_{1}^{\prime} & y_{1}^{\prime} & \ldots & \ldots & x_{n}^{\prime} & y_{n}^{\prime}
\end{array}\right] .
\end{aligned}
$$

The precision of the estimated parameters is the cofactor matrix, $Q_{\Delta \Delta}$, equals

$$
Q_{\Delta \Delta}=N^{-1} \text {. }
$$

The residual errors in, $\mathrm{x}$ and $y$, horizontal and vertical components of coordinate for each control point are computed based on

$$
\underset{2 n \times 1}{V}=\underset{2 n \times 1}{f}-\underset{2 n \times m}{B} \cdot \underset{m \times 1}{\Delta} .
$$

Thus, the residual vector for each position of common point is given by

$$
v_{\text {position }}=\sqrt{v_{x}^{2}+v_{y}^{2}} \text {. }
$$

The root mean square, RMS, of these errors is determined as follows

$$
R M S=\sqrt{\frac{V^{t} \cdot V}{2 n-m}} .
$$

A higher-order polynomials lead to a lower RMS but this does not mean that give a more accurate model. Highorder polynomials give more flexibility in bending the surface to fit the common stations. Furthermore, this bending may remarkably distort the coordinates of unknown points and add significant errors when the conversion is applied (Bolstad, 2016). Otherwise, the accuracy of the transformation model relies basically on the accuracy of the polynomial surface.

\section{Proposed software}

Information technologies are one of the most dynamically developing areas of science and engineering, which contributes to the improvement and continuity of new solutions, aimed at creating advanced information systems (Petrov, 2017). Automation of AutoCAD is an effective approach for time saving, raising the quality of processes and enhancing integration with other automated operations outside of AutoCAD (Lowe \& Hartman, 2011). The developed software in the current study, Figure 2, named “TopoGC" has been created in Visual C++ language. Moreover, to share information between this application and $\mathrm{Au}-$ toCAD a small program in Visual Basic was performed to generate an ActiveX control (TopoGCTransf.ocx).

The ActiveX control is extracted geometric information of entities (point, line, block, etc.) that is required in transformation process, after passing the values of polynomial coefficients. Figure 3 illustrates a flowchart of ActiveX control for automation of AutoCAD in Visual Basic.

The following are some lines of pseudo-code of AutoCAD automation:

Private Sub OpenCAD_Click (...) Dim acadApp As AcadApplication Dim acadDoc As AcadDocument

'Create a new AutoCAD instance acadApp $=$ New AcadApplicationClass () acadApp. Visible $=$ True acadApp.Application. WindowState $=$ acMin

'Open a new drawingacadDoc $=$ acadApp.Documents.Open (“[drawing name]”) acadDoc $=$ acadApp.ActiveDocument

End Sub

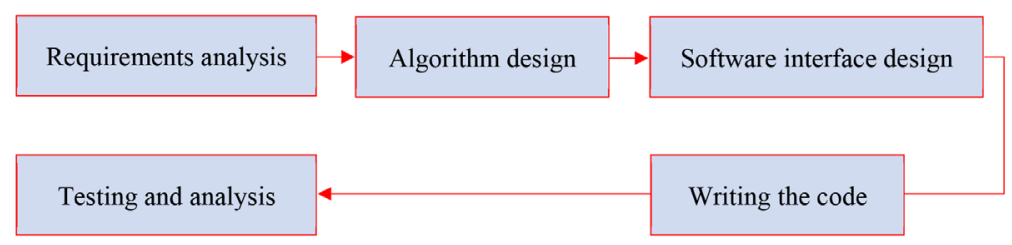

Figure 2. Schematic diagram of developed software life cycle

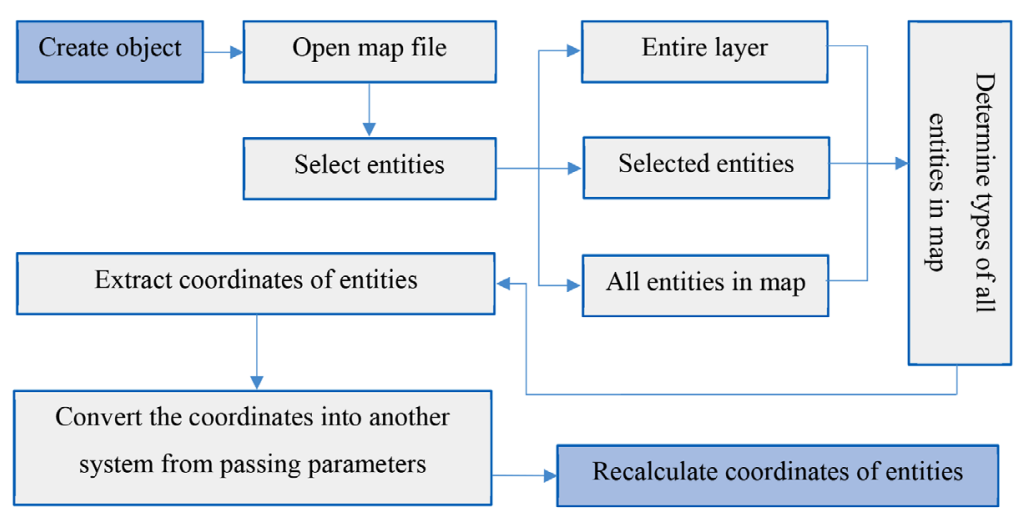

Figure 3. Flowchart of AutoCAD automation architecture 


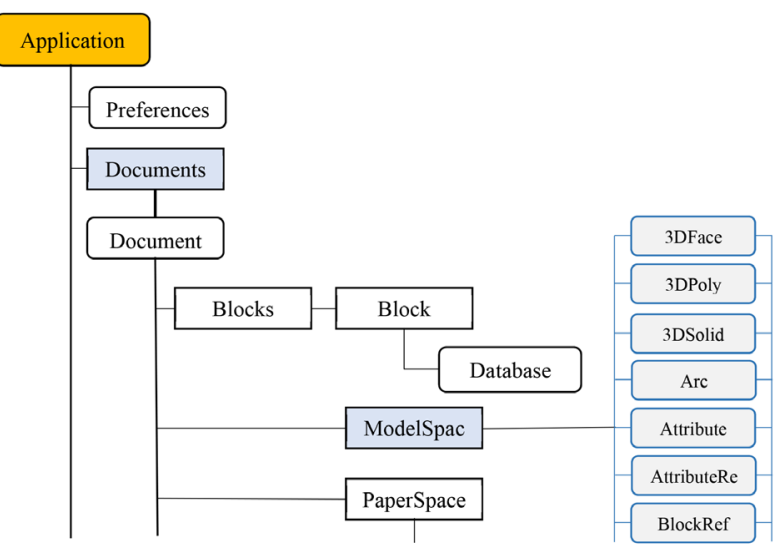

Figure 4. A small portion of AutoCAD Object Model

All the ActiveX objects can be exposed in one way for further programming and they are composed by hierarchies (Togores, 2019) as in Figure 4. The view of this hierarchical structure is referred to as the Object Model.

The system architecture consists of the following main components:

1) Determination of transformation parameters:

The dialogue box of transformation parameters computation is shown in Figure 5, where input data is rectangular coordinates of the common stations in both systems. These coordinates can be entered manually or imported from MS-Excel and can be reordered within the list. The software uses the least squares adjustment with a maximum number of control points that does not exceed hundred points. The output data are shown in a new window as a report contains the following:

- Degree of polynomial functions used in calculation.

- Coordinates of control point in two systems.

- Transformation parameters along with their standard deviations.

- Resulted residuals with respect to both systems.

- Standard deviation of unit weight.

2) Transformation of coordinates into a new system:

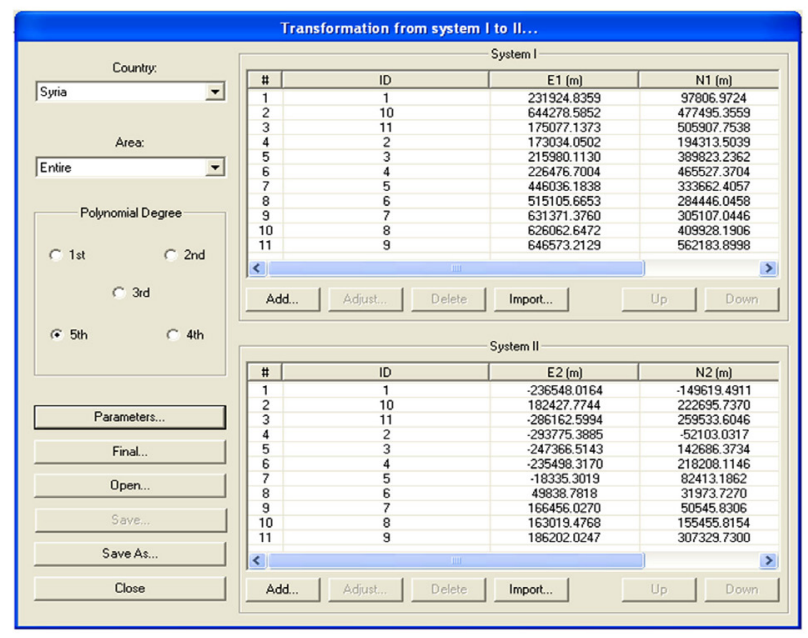

Figure 5. Transformation parameters window
The transformation of coordinates to another system from concluded parameters can be executed from the window which indicated in Figure 6, by implementing one of the following.

Inserting the coordinates of the point manually or from MS-Excel and then the coordinates are transformed into a new system. However, the transformed coordinates are displayed in the same list view and can be printed, exported to MS-Excel, or presented in AutoCAD drawing file.

Changing the coordinates of every entity in AutoCAD data by passing the transformation parameters and degree of polynomial to ActiveX control. The conversion between two coordinate systems in AutoCAD, can be done on selected objects, all objects or determined layer in DWG data.

Figure 7 shows the hierarchy chart of CTransformation2D and CFinal2Dcoord classes for transformation parameters calculation and conversion to the new system.

3) Solution of direct and inverse problems for projections

Figure 8 indicates a dialog box for calculating map projections, namely Lambert conformal, Transverse Mercator and stereographic mappings.

\section{Applied study and result}

To verify the proposed approach and its accuracy, in addition to test the developed software the following applied study has been performed to transform from Lambert to stereographic coordinates. The available data for this application is the coordinates of eleven first-order Syrian points indicated in Figure 9, where Syrian map is produced by using ArcGIS software. The rectangular coordinates in stereographic and Lambert projections are shown in Table 1.

The transformation parameters from Lambert to stereographic coordinates with their accuracies are calculated using developed software and listed in Table 2. The residuals at common stations are found to be at most subcentimeter. This attests to the high accuracy of the

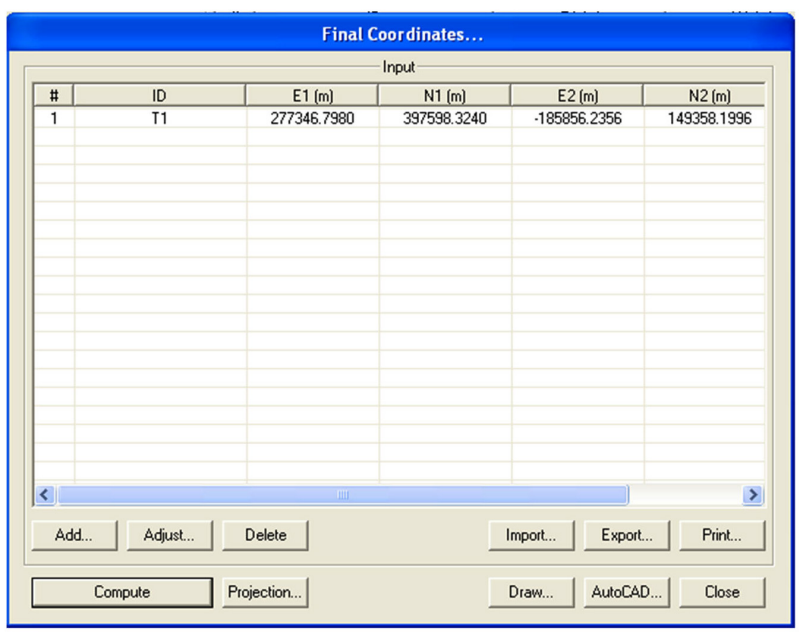

Figure 6. User interface of transformation to another system 

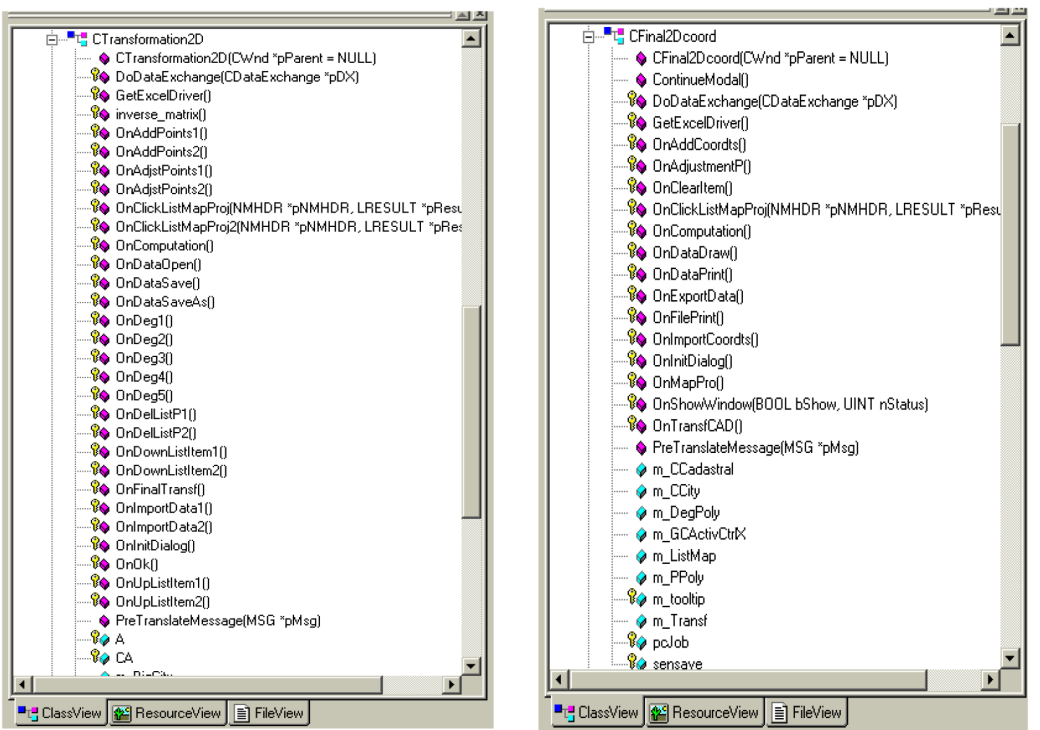

Figure 7. Hierarchy chart of CTransformation2D and CFinal2Dcoord Classes

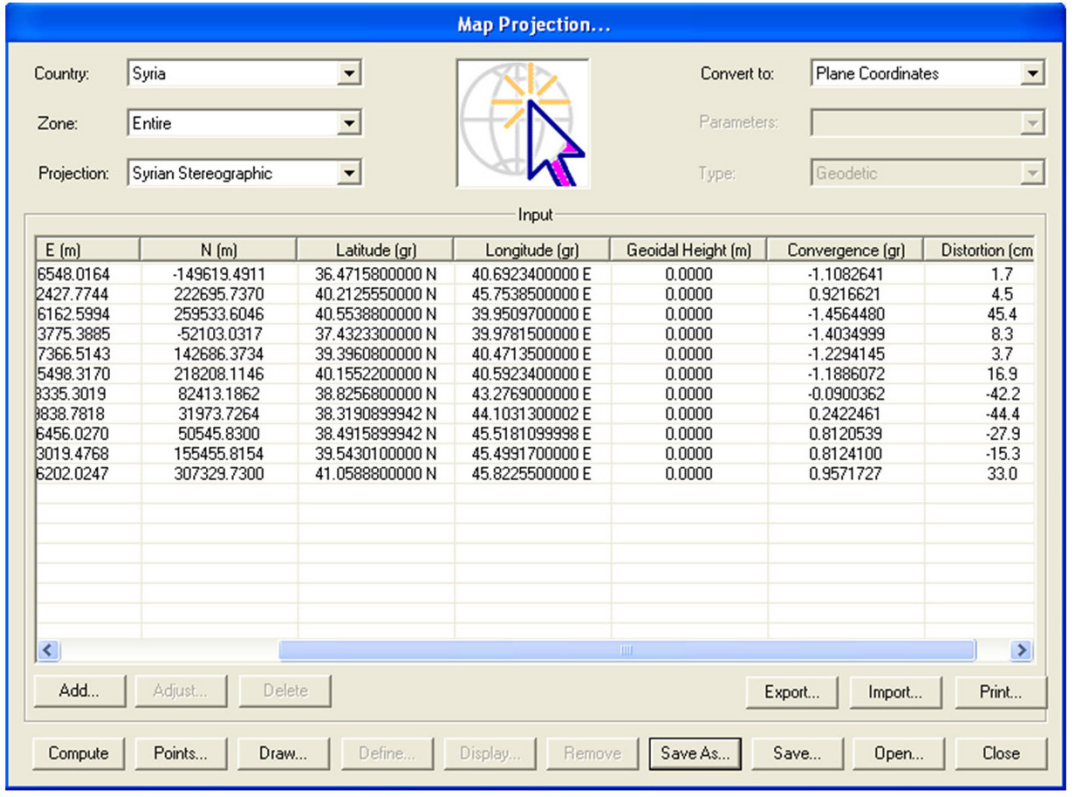

Figure 8. Window of calculating map projections

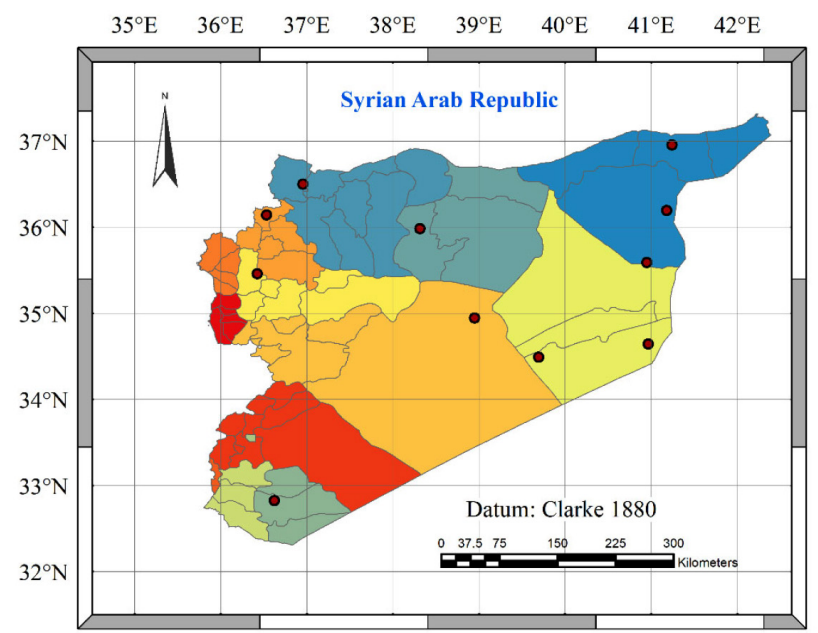

Figure 9. Distribution of common points 
Table 1. Projected coordinates of common points

\begin{tabular}{|c|c|c|c|c|}
\hline Point & $x_{S T}(\mathrm{~m})$ & $y_{S T}(\mathrm{~m})$ & $x_{L M}(\mathrm{~m})$ & $y_{L M}(\mathrm{~m})$ \\
\hline 1 & -236548.016 & -149619.491 & 231924.836 & 97806.972 \\
\hline 2 & -293775.388 & -52103.032 & 173034.050 & 194313.504 \\
\hline 3 & -247366.514 & 142686.373 & 215980.113 & 389823.236 \\
\hline 4 & -235498.317 & 218208.115 & 226476.700 & 465527.370 \\
\hline 5 & -18335.302 & 82413.186 & 446036.184 & 333662.406 \\
\hline 6 & 49838.782 & 31973.727 & 515105.665 & 284446.046 \\
\hline 7 & 166456.027 & 50545.831 & 631371.376 & 305107.045 \\
\hline 8 & 163019.477 & 155455.815 & 626062.647 & 409928.191 \\
\hline 9 & 186202.0247 & 307329.730 & 646573.213 & 562183.899 \\
\hline 10 & 182427.774 & 222695.737 & 644278.585 & 477495.356 \\
\hline 11 & -286162.599 & 259533.605 & 175077.137 & 505907.754 \\
\hline
\end{tabular}

proposed method and control points. Also, computing the coordinate differences at check points between the data and the reproduced from the derived transformation parameters performs another test on the accuracy of this model.

The following data were prepared in Syrian Lambert projection system using total station, the symbols and map composition such as a title, a scale, a legend and so on have been created. However, converting coordinates of unknown points in "TopoGC" can be done for inserted ones manually or imported from MS-Excel, Figure 7, or directly on AutoCAD drawings saved in DWG format, Figure 10.
Table 2. Coefficients of fifth degree polynomial and their accuracies

\begin{tabular}{|c|c|c|c|}
\hline Parameter & Value & $\begin{array}{c}\text { Standard } \\
\text { Deviation }\end{array}$ & Units \\
\hline$a_{0}$ & -470096.7122 & \pm 0.007 & $\mathrm{~m}$ \\
\hline$b_{0}$ & -243587.6819 & \pm 0.007 & $\mathrm{~m}$ \\
\hline$a_{1}$ & 1.0003773030 & $\pm 7.07 \times 10^{-8}$ & --- \\
\hline$b_{1}$ & $-1.5890020012 \times 10^{-2}$ & $\pm 7.07 \times 10^{-8}$ & --- \\
\hline$a_{2}$ & $-2.7900765202 \times 10^{-9}$ & $\pm 2.75 \times 10^{-13}$ & $\mathrm{~m}^{-1}$ \\
\hline$b_{2}$ & $-2.1038445198 \times 10^{-9}$ & $\pm 2.75 \times 10^{-13}$ & $\mathrm{~m}^{-1}$ \\
\hline$a_{3}$ & $1.9282043166 \times 10^{-15}$ & $\pm 5.26 \times 10^{-19}$ & $\mathrm{~m}^{-2}$ \\
\hline$b_{3}$ & $1.0262582815 \times 10^{-16}$ & $\pm 5.26 \times 10^{-19}$ & $\mathrm{~m}^{-2}$ \\
\hline$a_{4}$ & $-2.7901749985 \times 10^{-24}$ & $\pm 4.99 \times 10^{-25}$ & $\mathrm{~m}^{-3}$ \\
\hline$b_{4}$ & $-1.0951157002 \times 10^{-22}$ & $\pm 4.99 \times 10^{-25}$ & $\mathrm{~m}^{-3}$ \\
\hline$a_{5}$ & $-2.1026766897 \times 10^{-30}$ & $\pm 1.87 \times 10^{-31}$ & $\mathrm{~m}^{-4}$ \\
\hline$b_{5}$ & $1.8274376290 \times 10^{-31}$ & $\pm 1.87 \times 10^{-31}$ & $\mathrm{~m}^{-4}$ \\
\hline
\end{tabular}

\section{Conclusions}

The integration of spatial data between CAD system such as AutoCAD and GIS increases operational efficiency and enhances CAD mapping. The automation of AutoCAD is significant for map to map transformation to fulfil demands of AutoCAD users. Conformal conversion of rectangular coordinates using polynomial functions gives sufficiently accurate results for most purposes. The developed software relies on the least squares adjustment approach

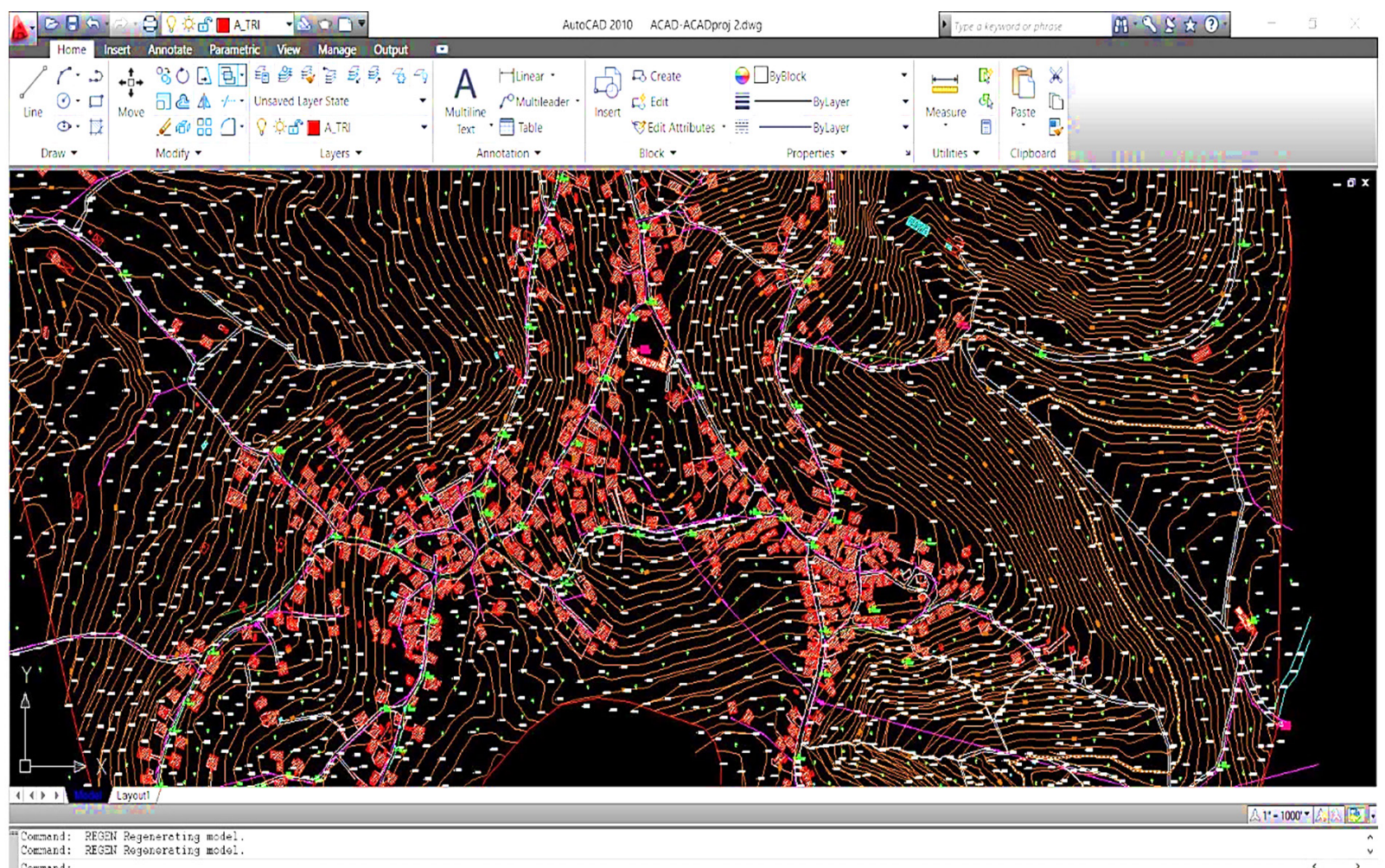

Figure 10. Transformation of Lambert coordinates to Stereographic ones of AutoCAD 
to perform corrected values of conversion parameters. The system architecture is split into two parts, the first one was written in Visual $\mathrm{C}++$ to compute conformal mappings and determine the polynomial coefficients, but the second one was built in Visual Basic to automate AutoCAD by creating ActiveX control. This study ensures that ActiveX control approach is reliable, accurate and efficient for automation of AutoCAD to change the coordinates of entities in DWG file.

\section{References}

Awange, J. L., \& Kiema, J. B. K. (2013). Fundamentals of GIS. In Environmental Geoinformatics (pp. 191-200). Springer, Berlin, Heidelberg. https://doi.org/10.1007/978-3-642-34085-7_13

Bolstad, P. (2016). GIS fundamentals: A first text on geographic information systems. Eider Press.

Burrough, P. A., McDonnell, R., McDonnell, R. A., \& Lloyd, C. D. (2015). Principles of geographical information systems. Oxford University Press.

Chrisman, N. (1999). A transformational approach to GIS operations. International Journal of Geographical Information Science, 13(7), 617-637. https://doi.org/10.1080/136588199241030

Deakin, R. E. (2004). A guide to the mathematics of map projections. School of Mathematical and Geospatial Sciences, RMIT University, Melbourne.

Esri, (2011). ArcGIS for AutoCAD 250. Technical Paper.

Greenfeld, J. S. (1997). Least squares weighted coordinate transformation formulas and their applications. Journal of Surveying Engineering, 123(4), 147-161. https://doi.org/10.1061/(ASCE)0733-9453(1997)123:4(147)

Janssen, V. (2009). Understanding coordinate reference systems, datums and transformations. International Journal of Geoinformatics, 5(4).

Herrault, P. A., Sheeren, D., Fauvel, M., Monteil, C., \& Paegelow, M. (2013). A comparative study of geometric transformation models for the historical "map of France" registration. Geographia Technica, (1), 34.

Karimi, H. A., \& Akinci, B. (2009). CAD and GIS integration. CRC Press. https://doi.org/10.1201/9781420068061
Kutoglu, H. S., \& Vaníček, P. (2006). Effect of common point selection on coordinate transformation parameter determination. Studia Geophysica et Geodaetica, 50(4), 525-536. https://doi.org/10.1007/s11200-006-0033-9

Maling, D. H. (1992). Coordinate systems and map projections. Pergamon Press. https://doi.org/10.1016/B978-0-08-037233-4.50020-0

Mikhail, E. M., \& Gracie, G. (1981). Analysis and adjustment of survey measurements. New York: Van Nostrand Reinhold.

Moreno, R., \& Bazán, A. M. (2017). Automation in the teaching of descriptive geometry and CAD. High-level CAD templates using script languages. In IOP Conference Series: Materials Science and Engineering, 245(6). IOP Publishing. https://doi.org/10.1088/1757-899X/245/6/062039

Pędzich, P. (2005). Conformal projection with minimal distortions. In XXII International Cartographic Conference Proceedings", La Coruna.

Petrov, M. N. (2017). Research into the methods of software product developing and maintaining. Sibirskij zhurnal nauki i tehnologi, 18(4).

Shi, M., Wang, X., Xin, W., Hui, G., Nan, M., Li, H., \& Song, S. (2016). AutoCAD map conversion based on teigha. NET and ArcGIS engine. DEStech Transactions on Engineering and Technology Research, (iect). https://doi.org/10.12783/dtetr/iect2016/3753

Snyder, J. P. (1987). Map projections - A working manual (Vol. 1395). US Government Printing Office. https://doi.org/10.3133/pp1395

Taşçi, L. (2009). The Adjustment of some geodetic networks using Microsoft excel solver. Turkish Journal of Science \& Technology, 4(2).

Thomas, P. D. (1952). Conformal projections in geodesy and cartography (Vol. 4). US Government Printing Office.

Togores, R. (2019). AutoCAD Expert's Visual LISP. Createspace Independent Publishing Platform.

Vanicek, P., \& Krakiwsky, E. J. (1987). Geodesy: the concepts. Elsevier.

Velsink, H. (2018). Testing methods for adjustment models with constraints. Journal of Surveying Engineering, 144(4), 04018009 . https://doi.org/10.1061/(ASCE)SU.1943-5428.0000260

Vincenty, T. (1987). Conformal transformations between dissimilar plane coordinate systems. Surveying and Mapping, 47(4), 271-274. 